\title{
Combating MRSA in Pakistan: An Integrated Drug Therapy Approach
}

\author{
Anas Rashid ${ }^{1,}$, , Usamah Rashid Qureshi ${ }^{2}$, Aiman Rashid ${ }^{3}$, Hamza Rashid $^{4}$ \\ ${ }^{1}$ Hamdard Institute of Pharmaceutical Sciences (HIPS), Hamdard University Islamabad Campus (HUIC), Islamabad, Pakistan \\ ${ }^{2}$ Department of Business Studies, Faculty of Economics and Business Studies, Pakistan Institute of Development Economics (PIDE), \\ Quaid-i-Azam University Campus, Islamabad, Pakistan \\ ${ }^{3}$ Department of Design and Manufacturing Engineering, School of Mechanical and Manufacturing Engineering (SMME), National \\ University of Sciences and Technology (NUST), NUST Campus, Islamabad, Pakistan \\ ${ }^{4}$ Department of Computer Sciences, Faculty of Natural Sciences, Quaid-i-Azam University (QAU), Islamabad, Pakistan
}

\section{Email address:}

anas.rashid_94@hotmail.com (A. Rashid), usamah04@yahoo.com (U. R. Qureshi), aiman.rashid@outlook.com (A. Rashid), hamza1886@gmail.com (H. Rashid)

\section{To cite this article:}

Anas Rashid, Usamah Rashid Qureshi, Aiman Rashid, Hamza Rashid. Combating MRSA in Pakistan: An Integrated Drug Therapy Approach. American Journal of Life Sciences. Vol. 3, No. 2, 2015, pp. 71-75. doi: 10.11648/j.ajls.20150302.13

\begin{abstract}
Clinical microbiologist are facing challenge to defeat the bacterial infections like Methicillin Resistant Staphylococcus aureus (MRSA) in humans; due to unique resistance in bacterial strain. There are limited medication options left to conquer this deadly toxicities, if holistic approach is ignored. Also the linked complications and consequences of infections may increase beyond threshold levels; if exercise only a single array of drug therapy. This empirical study aims to create synergy by adopting an integrated drug therapy approach for optimum treatment. This research article is based on primary microbial data collection (from sputum, urine and blood) in hospital; where the experiment focuses to investigate the highest risk vulnerable area. The result depicts high prevalence of MRSA in blood sample (50\%) among in-door patients of age above 50. The preventive and curative measures are discussed along with alternative multiple array of medication with prudent selection to achieve targeted and optimum outcome.
\end{abstract}

Keywords: S. aureus, MTCC 87, MRSA, Integrated Drug Therapy, Pakistan

\section{Introduction}

Currently, in advanced scientific era of this modern digital age, Microbiologists are observing a global hype in infectious human diseases through Staphylococcus aureus (S. aureus), which is precisely known as Methicillin Sensitive Staphylococcus aureus (MSSA). In this, infections of skin are due to the strains (i.e. invasive) of Methicillin Resistant Staphylococcus aureus (MRSA). It has a capacity to severely resist antibiotics; as experienced in the previous decades. These lethal infections are responsible for causing significant morbidity for mankind and stress (including both economic and financial) upon health care system. This study focuses on adopting an ingenious integrated approach to explore the effective solution by implementing preventive measures to control epidemic, using localized treatment by alternative medicines to cure and heal, and to treat the menace of human infections produced by MRSA.

\subsection{Challenges and Changes}

Today, controlling MRSA is still a clinical challenge faced by clinicians across the world. The objective of our study is to find out the economical, optimum and convenient methods to treat the human infections caused by MRSA [1]. Therefore, the focus of research is to consider alternative medicines (such as natural / herbal / ayurvedic, homeopathic, Chinese medicines) and modern therapeutic methods (such as radio waves, nuclear / radio-active / laser therapy) that are essential as well as effective; which not only boosts the natural physiological immune system but also efficiently diminish the infectious effects and therapeutic side effects.

Those, who are not involved in health care settings, are very often unaware of this silent and lethal epidemic. This lack of awareness comprises three folds which is; significance of risk and peril of hospital acquired MRSA 
infections, and their potential threat to overall health care system. In order to control the global menace of infections, a significant public behavioral change is needed. For this behavioral change to occur, a well-informed public is essential.

\subsection{Habitat}

As $S$. aureus is a highly contagious and ubiquitous bacterial species found throughout the ecosystem. Here the fatal entry of this microorganism starts from invading the skin (or deeper tissues); and later it multiplies to increase its population to cause a localized or systemic response, as in septicemia (blood poisoning), then a patient becomes infected. The hibernation or inactivated time period does not mean that it may not contaminate body area; but still it multiplies for its quantum population.

It is predominant in damp health-care environments; also in those persons who seem to be weaker, older and sicker than the over-all population that enhances the exposure of infection by weaker resistance (i.e. immunity). Many individuals either living or functioning together closely are the perfect congenial environment for the transmission of infectious diseases. It is reported that, individuals may carry this infection without having any visible indications.

\subsection{Causes}

Main cause of microbial resistance is due to the production of $\beta$-lactamase enzyme in the affected area [2]. Some strain of MRSA, termed as epidemic strains (EMRSA); however, sixteen EMRSA strain variants have been explored in the globe. In recent times, the common strain variants to affect hospitals are EMRSA fifteen and sixteen. After the development of infection, the choice of antibiotics, as efficient and effective remedy is reduced; although they are expensive and possibly show harmful side-effects towards the patient [3]. Recent evidence supports that household pets; such as cats, hens and dogs, can transmit MRSA to their owners [4]. The chart (Fig 1) below depicts the degree of risk factor sensitivity for bacterial growth:

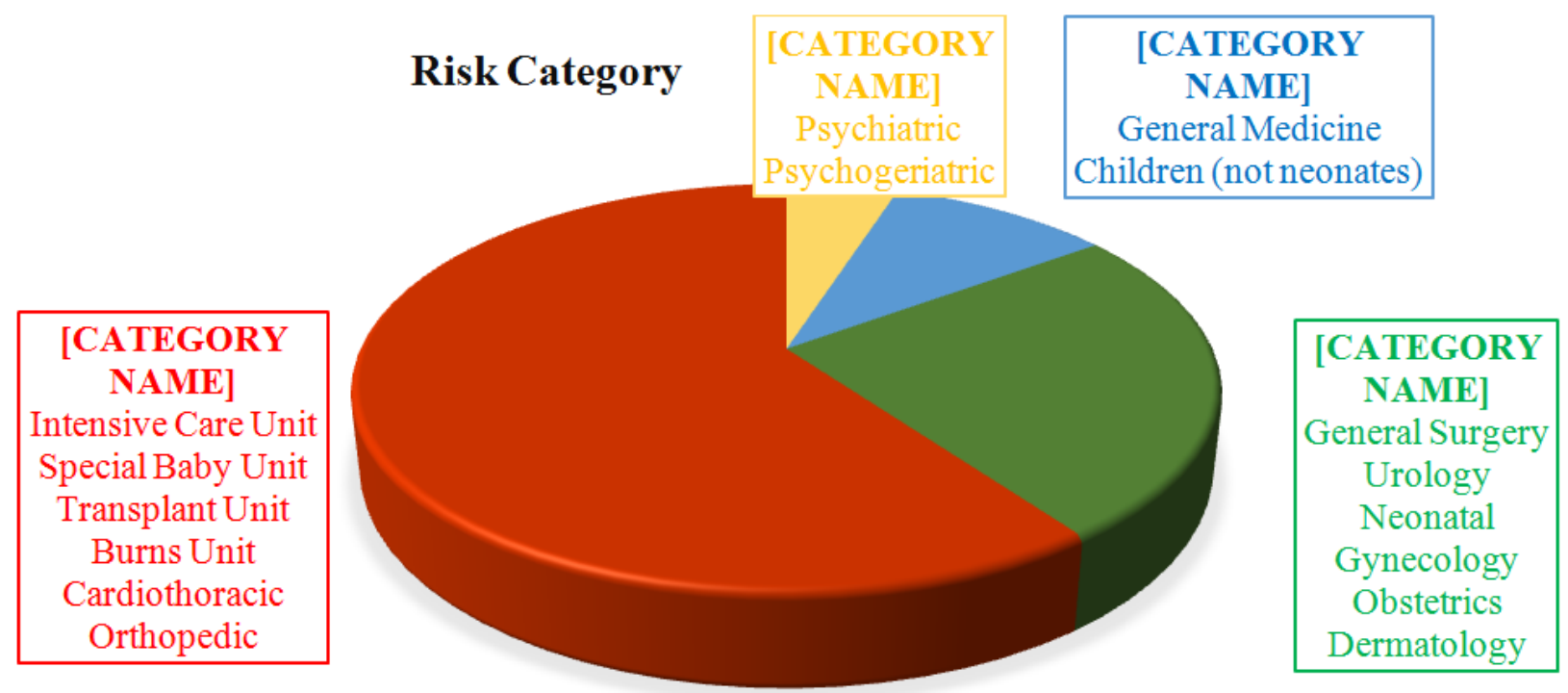

Fig. 1. The degree of risk factor sensitivity for bacterial growth.

\section{Material and Methods}

An empirical study was conducted from September to October 2014 at Holy Family Hospital, an 800 bed tertiary teaching hospital located in the Rawalpindi city of Pakistan. Firstly, we identified the patients and then the data (without the disturbance of routine work life) were gathered by using the information and support system from the targeted hospital. For this purpose, the collection of samples were gathered in containers which were already sterile by healthcare team using aseptic technique. The samples were transported without any delay for immediate processing.

Two media (Chocolate and Blood agar) were used for the identification and isolation of S. aureus [5]. Then, these collected samples were inoculated onto Chocolate agar as well as on Blood agar. The two tests used to identify the bacteria type i.e. coagulase and oxidase.
The Chocolate agar plates were incubated in a ten percent $(10 \%)$ carbon dioxide incubator at $37{ }^{\circ} \mathrm{C}$ for one day i.e. 24 hours [6]. The Blood agar plates were incubated for twenty four to thirty six hours $(24-36 \mathrm{hrs}$. $)$ at $37{ }^{\circ} \mathrm{C}$ [7]. The particular bacterial characteristics were identified by using standard microbiological techniques [8].

Methicillin Resistant Staphylococcus aureus was identified by using Vancomycin $(30 \mu \mathrm{g})$ and Gentamicin $(10 \mu \mathrm{g})$ disks [9]. Inducible resistance was identified in $S$. aureus by disk approximation test [10]. The data were documented and then critically analyzed for the completion of this study.

A reference strain, $S$. aureus MTCC 87, was used for standardization of antibiotic susceptibility testing.

\section{Results and Discussion}

872 total samples were processed from both in-patients 
and out-patients (Fig 2).

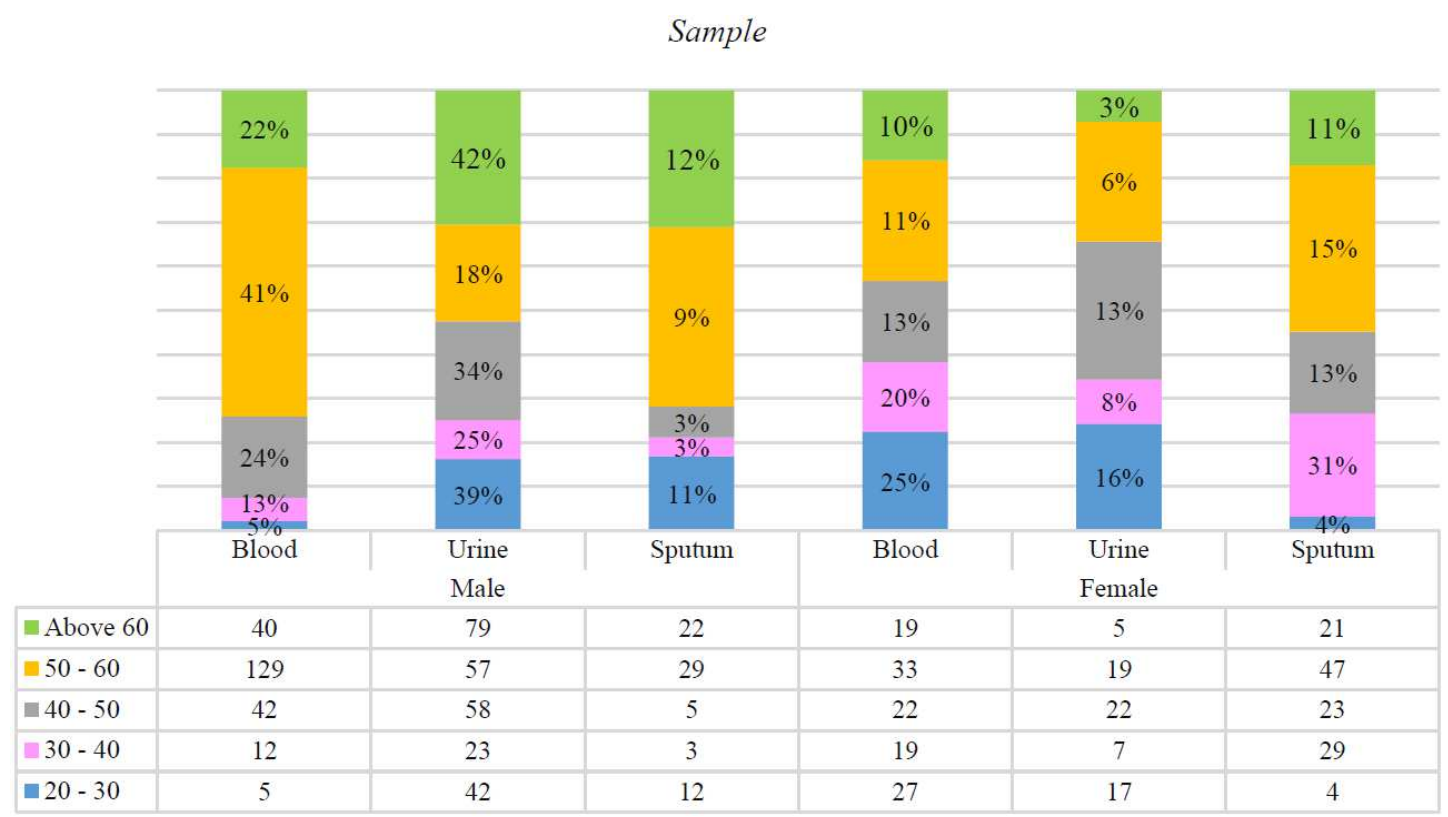

Fig. 2. Total Collected Samples - Segregated.

The highest rate of $S$. aureus from the processed samples was found in $50 \%$ blood samples; where 3/9 blood samples were males (age bracket in years: $50-60$ ) and 2/9 blood-samples were females (age bracket in years: $>60$ ) followed by $33.33 \%$ in urine; where each $2 / 6$ urine samples (age bracket in years: $50-60$ ) found both in males and females. However the lowest rate of $S$. aureus from the processed samples was found in sputum samples which is $16.66 \%$; where each $1 / 3$ sputum - samples found in males (age bracket in years: $50-60$ and $40-50$ ) and $1 / 3$ sputum samples found in females (age bracket in years: $30-40)$ (Fig 3).

S. aureus

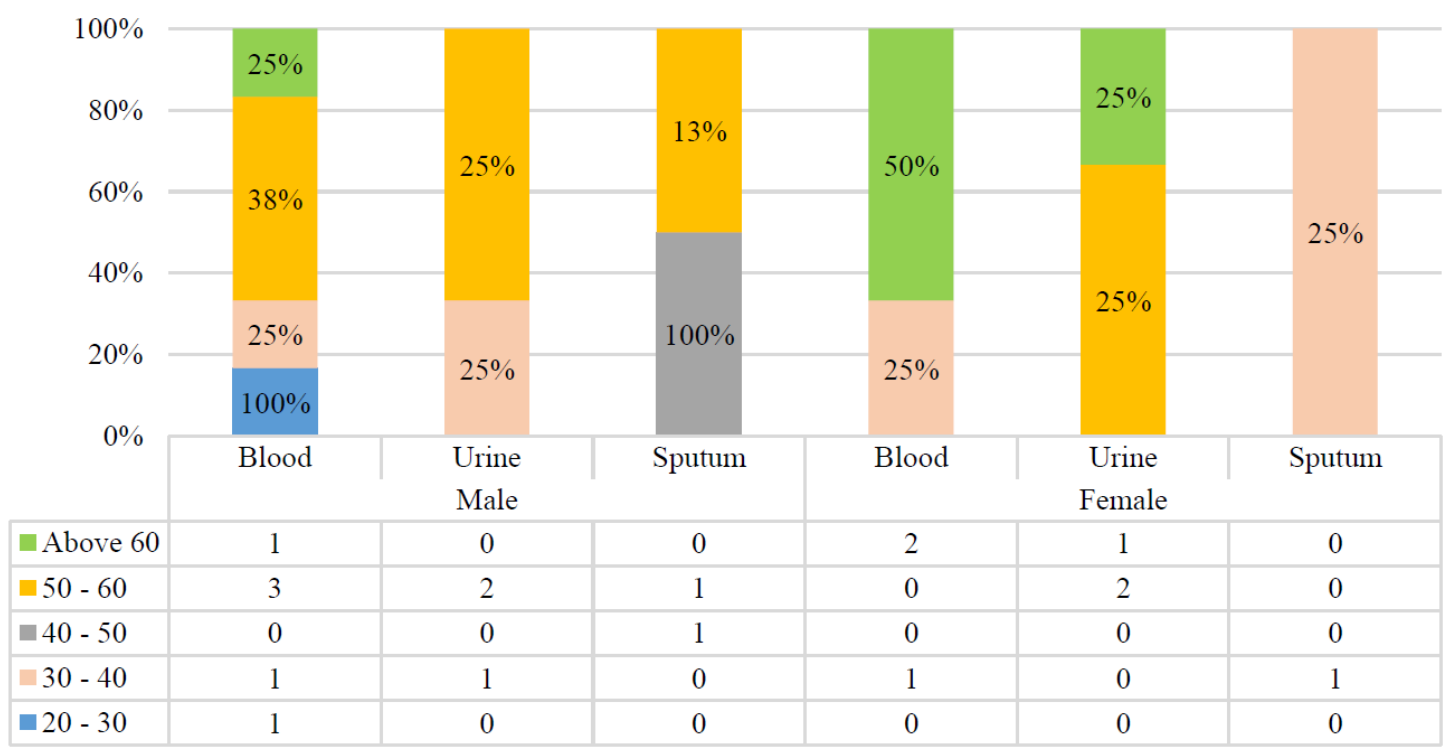

Fig. 3. Total Samples Contaminated with S. aureus - Segregated.

To conclude, the study showed high prevalence of MRSA among in-door patients.

\subsection{Preventive Measures (Economical and Accessible)}

The following preventive measures are essential to be taken to prevent early spread of infectious diseases:
Appropriate hand washing (including areas between fingers and nails) is generally accepted to be the first necessary action used for decreasing the infection. Alternatively, alcohol-hand-rub can be applied for decontamination [11]. Keep hand cleanliness - with each patient contact, replacing bed linen as well as bedding; after handling anything contaminated with body fluids, removing 
protective clothing / gloves; prior to aseptic technique, handling invasive devices and also before handling food. Also maintain cleanliness of general environment including atmosphere (as MRSA is both airborne and waterborne disease) [12]. Maintain an appropriate ratio of staff and patient. Keeping nails clean, short as well as polish free; avoid wearing wristwatches specifically finger-rings, is one of the best preventive tactical decision.

The above stated measures must be taken into account at all times and also among each and every individuals including persons engage with them in health care system.

\subsection{Curative Measures}

For mild skin infections, the use of either oral antibiotics or topical Mupirocin directed towards common skin flora, is the first line of treatment. For less severe infections, topical antibiotics are mostly useful [7]. However, MRSA strains are highly resistant to various antibiotics, including Cephalosporins, Macrolides and Quinolones.

The use of systemic antibiotic should be reserved for mild to moderate or severe infections that cover a large surface area. The use of an antimicrobial agent is highly recommended for the purpose of treatment. Antibiotics like Ciprofloxacin, Sulfamethoxazole / Trimethoprim, Linezolid, Rifampin or Vancomycin are right options for treatment. It is noticed that most of the infections with appropriate treatment resolved within ten days; if the symptoms not resolved then the right mix of antibiotics / medicines may be tried; that can simultaneously defend and destroy the bacterial invasion in the body and boost the self-immune system with least side-effects [13].

\subsection{Alternative Medicines}

Herbal drug treatment may be adapted such as turmeric (dried rhizome of Curcuma longa belonging to family Zingiberaceae), olive leaves extract, and essential oil, such as tea tree oil or lavender oil, black drawing salve, bentonite clay ichthammol ointment may be applied [14]. Some specialists advise washing the infected area with hydrogen peroxide and then applying the bentonite clay.

Selective range of electromagnetic waves / lasers and radio-nuclear therapy may be adopted as localized modern clinical treatment. MRSA was detected sensitive towards both solar (Ultraviolet A \& B) as well as germicidal (Ultraviolet $\mathrm{C}$ ) ultraviolet radiation ( $\mathrm{UV}-\mathrm{C}$ radiation is substantially more lethal). The calculated exposure of UV radiation (both solar and germicidal) increases the concentration of Sodium Chloride $(\mathrm{NaCl})$ medium; where cells exhibit increase in sensitivity [15].

The most common homeopathic treatment for MRSA may include Pulsatilla and Aconitum (genera in the family of Ranunculaceae); and also belladonna (species in the family of Solanaceae). Pulsatilla is a homeopathic remedy especially recommended in women and children. Aconitum is proven to be helpful in all kinds of the infection, including MRSA. In homeopathic doses, belladonna remedy (extremely small doses) is also used to cure the red skin, often associated with the infections.

\section{Conclusion}

As stated above, the study showed high prevalence of MRSA among in-door patients. However, the affected patients can socialize as usual; but proper decontamination of hands and use of face mask, need to be incorporated. To minimize the extent of infection, health-care-staff (including attendants and visitors) should make sure that they wash their hands with sterile liquid thoroughly between patient's clinical examinations.

The empirical results on human revealed that blood is the most vulnerable medium (or fluid) in adults (age in years < 50 years) for MRSA. Thus for blood transfusion and blood banking services; a systemic blood screening should be adopted. Health care associated infection (HCAI) should be highlighted prominently on the media at national and international level for public awareness and advocacy.

An integrated drug therapy approach (such as natural / herbal / ayurvedic, homeopathic, radio waves, Chinese medicines etc.) should be adopted in a strategic way to deplete the cause and effect, simultaneously to boost the natural physiological immune system tactically for targeted optimum outcome.

\section{References}

[1] McKenna, M. (2010). Superbug: The Fatal Menace of MRSA. Ed., pp. 288, Simon and Schuster, New York.

[2] Morello, J., Granato, P. and Morton, V. (2013). Lab Manual and Workbook in Microbiology: Applications to Patient care. 11th Ed., pp. 336, McGraw-Hill Education, New York.

[3] Weigelt, J.A. (2014). MRSA. 2nd Ed., pp. 240, CRC Press, London.

[4] Hektoen, L., Larsen, S., Ødegaard, S.A. and Løken, T. (2004). Comparison of Homeopathy, Placebo and Antibiotic Treatment of Clinical Mastitis in Dairy Cows Methodological Issues and Results from a Randomizedclinical Trial. Journal of Veterinary Medicine Series A, 51(910): 439-446.

[5] Hogg, S. (2013). Essential Microbiology. 2nd Ed., pp. 526, Wiley-Blackwell, Chichester, West Sussex.

[6] Cappuccino, J. and Sherman, N. (2013). Microbiology: A Laboratory Manual. 10th Ed., pp. 560, Pearson - Benjamin Cummings, Boston.

[7] Westh, H., Zinn, C.S., Rosdahl, V.T. and Sarisa Study, G. (2004). An International Multicenter Study of Antimicrobial Consumption and Resistance in Staphylococcus aureus Isolates from 15 Hospitals in 14 Countries. Microbial Drug Resistance, 10(2): 169-176.

[8] Brown, A. and Smith, H. (2014). Benson's Microbiological Applications: Laboratory Manual in General Microbiology, Complete Version. 13th Ed., pp. 576, McGraw-Hill Education, New York. 
[9] Smith, T.L., Pearson, M.L., Wilcox, K.R., Cruz, C., Lancaster, M.V., Robinson-Dunn, B., Tenover, F.C., Zervos, M.J., Band, J.D., White, E. and Jarvis, W.R. (1999). Emergence of Vancomycin Resistance in Staphylococcus aureus. New England Journal of Medicine, 340(7): 493-501.

[10] Tille, P.M. (2013). Bailey \& Scott's Diagnostic Microbiology. 13th Ed., pp. 1056, Elsevier, St. Louis.

[11] Aureden, K., Arias, K., Burns, L.A., Creen, C., Hickok, J., Moody, J., Oriola, S. and Risa, K. (2010). Guide to the Elimination of Methicillin-Resistant Staphylococcus aureus (MRSA) Transmission in Hospital Settings. 2nd Ed., pp. 65, APIC, Washington, DC.

[12] Pruthi, V. and Cameotra, S.S. (1997). Rapid identification of biosurfactant-producing bacterial strains using a cell surface hydrophobicity technique. Biotechnology Techniques, 11(9): 671-674.
[13] Ziakas, P.D., Zacharioudakis, I.M., Zervou, F.N. and Mylonakis, E. (2015). Methicillin-Resistant Staphylococcus aureus Prevention Strategies in the ICU: A Clinical Decision Analysis. Critical Care Medicine, 43(2): 382-393.

[14] Chan, B.C.-L., Bik-San Lau, C., Jolivalt, C., Lui, S.-L., Ganem-Elbaz, C., Paris, J.-M., Litaudon, M., Fung, K.-P., Leung, P.-C. and Ip, M. (2011). Chinese Medicinal Herbs Against Antibiotic resistant Bacterial Pathogen. Science against Microbial Pathogens: Communicating Current Research and Technological Advances, 2(3): 773-781.

[15] Whitmont, R.D. (2011). MRSA and CAM. http://www.homeopathicmd.com/2011/04/mrsa-and-cam/. Accessed 08 October 2014. 